\title{
DERECHO Y TECNOLOGÍA MÉDICA: ORÍGENES DE SU LEGISLACIÓN EN ARGENTINA
}

\author{
Alvaro Monzón Wyngaard,1 Jorge E. Monzón,2 Agustín S. Carlevaro,3 \\ Patricio Monzón Battilana, 4 Victoria Monzón 5 y Matias Payes6
}

\section{Introducción}

La evaluación de tecnología sanitaria, la difusión de las innovaciones y su transferencia, los factores que conllevan a una rápida aceptación de las mismas y el rol que cumple la tecnología en la sociedad delimitan el campo de los fenómenos ciencia, técnica y salud. Su reconocimiento en los sistemas jurídicos a través de la instauración de un proceso para la incorporación de las mismas dentro de las esferas del Estado, más precisamente, en sus sistemas sanitarios, cuenta con cuatro etapas, a saber, a) planificación, b) evaluación, c) adquisición $\mathrm{y}, \mathrm{d})$ gestión. En este trabajo, desarrollamos los orígenes del segundo y tercer proceso, lo que en doctrina internacional y las prácticas de los gobiernos se denomina "evaluación de tecnologías sanitarias e incorporación" de éstas a los sistemas de salud, ya que consideramos que estudiar estos fenómenos desde su perspectiva histórica y legal, coadyuvaría a un mejor desempeño de nuestros Estados en pos del bienestar general y las inversiones que se realicen al respecto, determinando qué órgano es el competente para realizarlo.

Sosteníamos, en una publicación anterior, ${ }^{7}$ que según el Office of Technology Assessment (OTA) de los Estados Unidos, la Tecnología Médica está

${ }^{1}$ Profesor Titular de la Cátedra "B" de Derecho Público Provincial y Municipal, Facultad de Derecho y Ciencias Sociales y Políticas-UNNE. UNNE.

${ }^{2}$ Profesor Titular y Director del Grupo de Ingeniería Biomédica de Investigación. FACENA-

${ }^{3}$ Becario CONICET. Adscripto de la Cátedra "B" de Derecho Público Provincial y Municipal, Facultad de Derecho y Ciencias Sociales y Políticas-UNNE.

" Jefe de Trabajos Prácticos de la Cátedra "B" de Sociología. Facultad de Derecho y Ciencias Sociales y Políticas-UNNE.

${ }^{5}$ Becaria SECYT-UNNE. Adscripta de la Cátedra "B" de Derecho Agrario y de Minería. Facultad de Derecho y Ciencias Sociales y Políticas-UNNE.

${ }^{6}$ Becario Pre-Grado SECYT-UNNE. Ayudante Alumno de la Cátedra "A" de Derecho Político. Facultad de Derecho y Ciencias Sociales y Políticas-UNNE.

${ }^{7}$ Álvaro Monzón Wyngaard y otros. "Tecnología Médica y Derecho: La Potencialidad de la Ingeniería Biomédica". En: Revista de la Facultad de Derecho y Ciencias Sociales y Políticas de la Universidad Nacional del Nordeste. Corrientes: ConTexto, Nueva Serie. Año 10-N 18 , 2016; p. 63 y ss. 
conformada por: los medicamentos, los aparatos, los procedimientos médicos y quirúrgicos utilizados en la atención médica y los sistemas organizativos con los que se presta la atención sanitaria. Por lo tanto, la tecnología médica no sólo es máquinas o medicamentos, sino también la propia práctica clínica y el modo en que ésta se organiza. ${ }^{8}$ Cabe destacar que estas nuevas tecnologías no se limitan a tratar de curar el daño o la enfermedad orgánicos - farmacéuticos-, tampoco a mejorar la salud, como es el caso de los regímenes alimenticios -nutricionistas-o los programas orientados a lograr un buen estado físico - kinesiólogos-, sino que cambian aquello en lo que consiste ser un organismo biológico haciendo posible refigurar los procesos vitales mismos con el fin de maximizar su funcionamiento y mejorar sus resultados — médicos e ingenieros biomédicos - dando origen a una resignificación del concepto de persona.

\section{Desarrollo}

Los autores en general identifican el inicio de la Tecnología en Medicina con dos momentos claramente diferenciados durante el Siglo XIX, ambos, asociados al diagnóstico médico. El primero hacia el año 1816: la invención del estetoscopio marca el hito tecnológico, con un fuerte componente ético, de lo que entendemos por tecnología médica. Efectivamente hasta 1816 los procedimientos de diagnóstico eran muy limitados y la forma habitual de obtener información por parte del médico consistía en aplicar la oreja a la caja torácica y escuchar los latidos del corazón.

En el mencionado año el médico francés René-Théophile-Hyacinthe Laennec tuvo la necesidad de auscultar a una joven dama pasada en carnes:

Comprendió que sería imposible a causa de la barrera que oponían los pechos, y tratar de levantar o separar éstos para facilitar la auscultación hubiera sido una indelicadeza. (...) En un momento de inspiración, enrolló un cuaderno y colocó uno de los extremos del tubo así formado entre los pechos de la paciente, y el otro extremo lo aplicó a su oreja. Tuvo la satisfacción de comprobar que los latidos eran realmente más audibles que si hubiera apoyado la oreja sobre el esternón. Luego se proveyó de tubos de madera, con lo que inventó el estetoscopio (de las palabras griegas que significan "ver el pecho"). ${ }^{9}$

${ }^{8}$ Tecnología "Salud". (Consulta del 29/IX/15). Disponible: [http://socytec2012ng6.blogspot. com.ar/2012/05/tecnologia-salud.html].

${ }^{9}$ Isaac Asimov. Historia y Cronología de la Ciencia y los Descubrimientos. Cómo la ciencia ha dado forma a nuestro mindo. Barcelona-España: Ariel. 2014 
Michel Foucault, en su libro El Nacimiento de la Clínica. Una Arqueología de la mirada médica, magistralmente sentencia:

El estetoscopio, distancia solidificada, transmite acontecimientos profundos e invisibles a lo largo de un eje medio táctil, medio auditivo. La mediación instrumental en el exterior del cuerpo autoriza un retroceso que mide una distancia moral; la prohibición de un contacto físico permite fijar la imagen virtual de lo que ocurre lejos y por debajo de la región visible. La lejanía del pudor es, para lo oculto, una pantalla de proyección. Lo que no puede verse se muestra en la distancia de lo que no se debe ver. La mirada médica, así armada, más que pronunciarlo, envuelve el concepto de "mirada". Comprime en una estructura única campos sensoriales diferentes. ${ }^{10}$

El segundo momento está dado por el descubrimiento de los Rayos X hacia 1895. Por cuestiones del azar, el alemán Wilhelm Roentgen, utilizando una pantalla cubierta de sales de bario y a más de un metro de la emisión de los rayos catódicos, observó rayos desconocidos. Decidido a atravesar con los mismos una estructura humana, utilizó la mano izquierda de su esposa Bertha y quedó sumamente sorprendido al comprobar la imagen nítida de los huesos de la mano, lo que se considera como la primera radiografía de la historia. ${ }^{11}$

Gracias a sus comprobaciones, Roentgen, es considerado como el artífice de la segunda Revolución científica.

A éstos, se agregan otros acontecimientos científicos. Siguiendo al citado Isaac Asimov podemos plantear la siguiente Cronología de la Tecnología Médica, a saber: Invención del Electrocardiograma (1903); Desarrollo de la Quimioterapia (1907); Desarrollo de la ultracentrifugadora (1923); Desarrollo de la técnica de la resonancia magnética (1938); Introducción de la cateterización cardíaca (1941); Diseño de un riñón artificial (1945); Adopción del pulmón artificial (1953); Desarrollo del marcapaso de pequeño tamaño (1957); e Implantación de un corazón artificial (1969), entre otros.

\footnotetext{
${ }^{10}$ Michel Foucault. El Nacimiento de la Clínica. Una arqueología de la mirada médica. Buenos Aires: Siglo XXI editores; 2014.

${ }^{11}$ Pedro Gargantilla. Breve Historia de la Medicina. Del Chamán a la Gripe A. Madrid: Ediciones Nowtilus, S.L.; 2011.
} 
208 Álvaro Monzón Wyngaard, Jorge E. Monzón, Agustín S. Carlevaro, Patricio Monzón Battllana, Victoria Monzón y Matías Payes

\section{Argentina}

Originariamente la protección de la Salud Individual no incumbía al Estado, razón por la cual éste no apostaba a su reglamentación salvo que existiera un flagelo que atente contra la lozanía de todos los habitantes. Así, nos encontrábamos con tres instituciones que bregaban por la Salud, por un lado la Asociación de Socorros Mutuos; por otro la Comunidad Religiosa y; en tercer lugar la municipalidad de Buenos Aires. Con el modelo liberal tampoco el Estado se involucra, recién con el advenimiento del Constitucionalismo Social el Estado comienza a ingerirse en la reglamentación de este derecho. Sin embargo, pasaremos revista de los hitos más importantes que permitieron establecer las bases de nuestro sistema público de salud actual intervencionista.

A finales del siglo XIX y comienzos del XX la medicina era familiar, las prácticas médicas se llevaban a cabo en la propia casa del enfermo al cual concurría el profesional con su clásico maletín con los insumos necesarios para elaborar su diagnóstico, así como el consultorio también se encontraba generalmente establecido en una de las habitaciones de la propia casa del galeno.

Cuando se requería de prácticas un poco más complejas se concurría al sanatorio o al hospital, pero incluso entonces, el médico era quien tenía el poder de decisión sobre el tratamiento y su efectiva aplicación.

Hacia 1910 los hospitales municipales de Buenos Aires se caracterizaban por la escasez de recursos técnicos, asociados —además- a una débil tecnologización de la atención hospitalaria. ${ }^{12}$

Diez años más tarde se registra en Buenos Aires el aumento de instalaciones de internación vinculadas a especialidades (v. gr. enfermedades de pulmón, clínica obstétrica o bien clínica ginecológica) y "las de tecnología de diagnóstico y tratamiento". Siguiendo esta inteligencia, se registra mayor gasto en aparatología y otros insumos. Estas pautas son reconocibles en los servicios públicos, convirtiendo de esta manera al hospital público en el lugar donde el médico continúa su formación y dispone de los recursos técnicos necesarios para la atención de los pacientes de su comunidad, entre éstos, su clientela privada.

En el ámbito del mutualismo ocurre algo similar. Se halló el aumento sostenido -así como su costo- de prácticas vinculadas a la "nueva" tecnología

\footnotetext{
${ }^{12}$ Susana Belmartino. La Atención Médica Argentina en el Siglo XX. Instituciones y Procesos. Buenos Aires: Siglo XXI Editores, Argentina; 2006.
} 
de ese momento. Así lo refiere La Mutualidad, XXXVIII5, de 1939, citada por Belmartino:

Los progresos técnicos actuales exigen, por ejemplo, para el mejor diagnóstico, radiografías seriadas, especialmente para las enfermedades del aparato digestivo, radiografias practicadas previa preparación del enfermo con métodos de contraste (broncografia, pielgrafía, colescitografia, ventriculografia, etc.) radiografias de frente y de perfil para tórax, y en ciertos casos realizadas periódicamente, etc. En parangón con los nuevos tratamientos fisioterápicos (radioterapia, diatermia, ondas cortas, rayos ultravioleta, rayos infrarrojos, etc.) fue necesaria la adquisición de nuevos aparatos. (...) Una serie de métodos para clínicos, multiplicados en estos últimos años han acarreado nuevos gastos. Así, para el diagnóstico de las enfermedades del corazón debe recurrirse al "electrocardiógrafo", para el estudio funcional de la glándula tiroides debe solicitarse el "metabolismo basal", para ciertos exámenes del sistema nervioso debe realizarse "electrodiagnóstico", etc. ${ }^{12}$

Si bien el ícono de la modernización tecnológica fueron los rápidamente generalizados Rayos X, el crecimiento sostenido de otros métodos alternativos de diagnóstico obligó a repensar la propia estructura de la sanidad pública. Efectivamente al constituirse el Ministerio de Salud Pública de la Nación bajo la conducción de Ramón Carrillo, el Reglamento Interno (Parte General, Organigrama 2), ya preveía una Subsecretaría Técnica de la que dependían varias Direcciones, entre ellas, la Dirección de Medicina Tecnológica. ${ }^{13}$

Carrillo estableció un orden de prelación de las problemáticas que tenían prioridad en el tratamiento ministerial, ubicando a "Las deficiencias de locales, de instrumentos y equipos" ya entre las más acuciantes. ${ }^{13}$

En este devenir hemos dejado para el final la aproximación jurídica. No hemos encontrado referencias en la legislación argentina en el siglo XIX, ni siquiera de franquicias aduaneras para la importación de equipos ni tampoco promociones (como sí las hubo para la importación de equipamiento tecnológico en astronomía y minería).

12 Susana Belmartino. La Atención Médica Argentina en el Siglo XX. Instituciones y Procesos. Buenos Aires: Siglo XXI Editores, Argentina; 2006.

${ }^{13}$ Karina Ramacciotti. La política sanitaria del peronismo. Buenos Aires: Biblos; 2009. 


\section{0 Álvaro Monzón Wyngaard, Jorge E. Monzón, Agustín S. Carlevaro, Patricio Monzón Battilana, Victoria Monzón y Matías Payes}

A fin de comprender las condiciones necesarias para establecer este proceso evaluativo y de adquisición, consideramos de menester importancia analizar el estado del derecho de la salud, derecho administrativo y el (no conocido en ese entonces) derecho biomédico argentino. Para la consecución de tal objetivo, expondremos una serie de normas que reúnen competencias básicas que corresponden a la propia esfera de evaluación y compra/adquisición, por distintas formas jurídicas, de insumos y equipamiento hospitalario, las cuales se hallan diseminadas en cada uno de los sistemas jurídicos en dos o más cuerpos de normas respectivamente, a fin de establecer un orden lógico por el cual un producto médico debía atravesar. Para tal examen, basados en criterios que responden a manifestaciones similares en los países latinoamericanos, tomamos como unidades de análisis a nuestro país, destacando elementos que fueron extraídos de los fenómenos económicos, administrativos y sociales dados en la historia de la legislación, como también datos del presente que explican el estado actual del sistema sanitario.

Las primeras regulaciones son del siglo $\mathrm{XX}$, primera centuria, en la que propiciamos este esquema. $^{14}$

\begin{tabular}{|c|c|c|c|}
\hline $\begin{array}{l}\text { Importación de } \\
\text { productos }\end{array}$ & $\begin{array}{l}\text { Régimen } \\
\text { impositivo }\end{array}$ & $\begin{array}{l}\text { Equipamientos } \\
\text { radiológicos }\end{array}$ & $\begin{array}{c}\text { Contralor de } \\
\text { productos }\end{array}$ \\
\hline $\begin{array}{l}\text { Año 1904. Ley N } \\
4495 \text { de Instru- } \\
\text { mental para la } \\
\text { oficina de médicos } \\
\text { de la Policía de la } \\
\text { Capital }\end{array}$ & $\begin{array}{l}\text { Año 1935. Decreto } \\
\text { N}^{\circ} 64236 \text { del } 30 \\
\text { de junio. Regla- } \\
\text { mento General } \\
\text { de impuestos } \\
\text { internos; título } \\
\text { VIII, artículos de } \\
\text { uso higiénico, de } \\
\text { tocador, afeites, } \\
\text { perfumes, aguas } \\
\text { minerales, especia- } \\
\text { lidades medicina- } \\
\text { les y termómetros } \\
\text { clínicos. }\end{array}$ & $\begin{array}{l}\text { Año } 1928 \text {. Ley No } \\
11537 \text { de Adqui- } \\
\text { sición de material } \\
\text { para el Instituto de } \\
\text { Radiología y Fisio- } \\
\text { terapia de Buenos } \\
\text { Aires. }\end{array}$ & $\begin{array}{l}\text { Año } 1943 \text {. Decreto } \\
\mathrm{N}^{\circ} 10317 \text { del } 8 \text { de } \\
\text { octubre de Comi- } \\
\text { sión Nacional de } \\
\text { arancel y contralor } \\
\text { de productos medi- } \\
\text { cinales: dependen- } \\
\text { cia del Departa- } \\
\text { mento Nacional de } \\
\text { Higiene. }\end{array}$ \\
\hline
\end{tabular}

${ }^{14}$ Anales de Legislación Argentina. Repertorio Años 1852-1976. Tomos I y II. Buenos Aires: La Ley; 1978. 


\begin{tabular}{|c|c|c|}
\hline $\begin{array}{l}\text { Año } 1942 \text {. Decreto } \\
110.061 \text { del } 30 \text { de } \\
\text { enero de Derechos } \\
\text { aduaneros: entrada } \\
\text { temporal de radio } \\
\text { y rayos x, para su } \\
\text { reparación. }\end{array}$ & $\begin{array}{l}\text { Año } 1942 \text {. Decreto } \\
110.061 \text {, antes } \\
\text { consignado en } \\
\text { la columna de } \\
\text { "Importación de } \\
\text { productos medici- } \\
\text { nales". }\end{array}$ & $\begin{array}{l}\text { Año } 1944 \text {. Decreto } \\
\mathrm{N}^{\circ} 29.671 \text { del } 30 \\
\text { de octubre de De- } \\
\text { terminación y con- } \\
\text { tralor de materiales } \\
\text { críticos (Ratificado } \\
\text { por Ley } N^{\circ} 13892 \text {; } \\
\text { ampliado por } \\
\text { Decreto } 1143 / 45 \text { y } \\
\text { derogado parcial- } \\
\text { mente por Decreto } \\
5201 / 48 \text { ). }\end{array}$ \\
\hline \multirow[t]{3}{*}{$\begin{array}{l}\text { Año } 1946 . \text { Ley } \\
\mathrm{N}^{\circ} 12922 \text { de } \\
\text { Ratificación del } \\
\text { Decreto-Ley } \\
\text { sobre impuestos y } \\
\text { aduana: Decreto } \\
\mathrm{N}^{\circ} 15364 / 46 \text { de } \\
\text { Liberación de de- } \\
\text { rechos aduaneros a } \\
\text { materiales sanita- } \\
\text { rios: exclusiones. }\end{array}$} & $\begin{array}{l}\text { Año } 1943 \text {. Decreto } \\
\mathrm{N}^{\circ} 149.712 \text { del } \\
12 \text { de mayo de } \\
\text { Requisitos para las } \\
\text { categorías de mé- } \\
\text { dicos radiólogos y } \\
\text { de laboratoristas, } \\
\text { rentados con fon- } \\
\text { dos del subsidio } \\
\text { nacional. }\end{array}$ & $\begin{array}{l}\text { Año } 1947 \text {. Decreto } \\
29112 \text { de Régimen } \\
\text { para la aprobación } \\
\text { de las especialida- } \\
\text { des medicinales. }\end{array}$ \\
\hline & $\begin{array}{l}\text { Año } 1943 \text {. Decreto } \\
\text { N}^{\circ} 5482 \text { del } 16 \text { de } \\
\text { agosto de Precios } \\
\text { máximos para } \\
\text { películas radiográ- } \\
\text { ficas. }\end{array}$ & $\begin{array}{l}\text { Año 1947. Decreto } \\
\mathrm{N}^{\circ} 40.185 \text { del } 20 \\
\text { de diciembre de } \\
\text { Funcionamiento } \\
\text { de los laboratorios } \\
\text { de análisis clínicos } \\
\text { aplicados a la } \\
\text { medicina. }\end{array}$ \\
\hline & $\begin{array}{l}\text { Año } 1944 \text {. De- } \\
\text { creto } 1192 / 44 \text { de } \\
\text { Expropiación de } \\
\text { elementos para el } \\
\text { catastro radiológi- } \\
\text { co, modificado por } \\
\text { Decreto } 31362 / 44\end{array}$ & $\begin{array}{l}\text { Año 1949. Decreto } \\
\mathrm{N}^{\circ} 25209 \text { del } 10 \\
\text { de octubre de Con- } \\
\text { tralor de la venta } \\
\text { de materiales, } \\
\text { equipos, aparatos } \\
\text { e instrumental de } \\
\text { uso médico. }\end{array}$ \\
\hline
\end{tabular}




\section{2 Álvaro Monzón Wrigaard, Jorge E. Monzón, Agustín S. Carlevaro, Patricio Monzón Battilana, Victoria Monzón y Matías Payes}

Uno de los datos científicos más significativos que hemos hallado en nuestra investigación es el Decreto $\mathrm{N}^{\circ} 25209$ del 10 de octubre de 1949 que crea el Ministerio de Salud Pública y Previsión Social, organismo encargado de controlar la aplicación y venta de materiales, equipos, aparatos e instrumental de uso médico (Boletín Oficial del 18-X-1949). ${ }^{15}$

La urgencia de esta nueva normativa está basada en las normas establecidas por el Decreto $N^{\circ} 29112 / 47$ que regula la aprobación de las especialidades medicinales, y en las siguientes taxativas consideraciones:

El empleo de ciertos aparatos de uso médico ha originado, por causas dificiles de establecer, desgraciados accidentes a algunas personas sometidas a la utilización de dichos aparatos; $y$,

Para prevenir la repetición de tales accidentes es necesario disponer que ningún aparato de uso médico pueda ser vendido en el país sin previo contralor de carácter técnico que establezca las condiciones de su utilización en la práctica, -el subrayado es nuestro- de modo que no se convierta en factor peligroso para la integridad física de los médicos y los pacientes.

En consecuencia del citado Decreto No 25209/49 (firmado por Perón y Ramón Carrillo) se coligen los siguientes resultados:

$\left.1^{\circ}\right)$ El Ministerio de Salud Pública es el único y excluyente organismo encargado de controlar la "aplicación" y la "venta" en nuestro país de materiales, equipos, aparatos e instrumental de uso médico;

$2^{\circ}$ ) Cualquier producto de uso médico antes referenciado, indispensablemente debe estar certificado para proceder a su venta;

$3^{\circ}$ ) Se faculta al Ministerio a inspeccionar y reglamentar, en especial referencia a los aparatos, equipos o instrumentos médicos que ya estén en funcionamiento;

$4^{\circ}$ ) Disponer multas (de 500 a 10.000 pesos moneda nacional) o la clausura de los respectivos locales, en caso de infracciones.

\footnotetext{
${ }^{14}$ Anales de Legislación Argentina. 1949. Tomo IX-A. Buenos Aires: Editorial La Ley, 1950.
} 


\section{Conclusiones}

Los primeros en adoptar y difundir tecnología médica en sus prácticas no fueron los hospitales públicos sino los galenos establecidos en las ciudades, que operaban en forma privada a través de una vigilancia continua a sus pacientes, sustentada en sus conocimientos y habilidades clínicas y producían un estrecho vínculo de confianza. Sin embargo, algunas de las tecnologías alternativas utilizadas, a pesar de que eran aplicadas a la población, tenían poca utilidad.

Frente a esa vulnerabilidad, basada en el modelo médico hegemónico, el Estado progresivamente fue involucrándose en el control y luego vigilancia de la tecnología médica.

En su plano organizacional, en el Estado Argentino se contaba con unidades descentralizadas para asegurar el goce de este derecho a través del acceso equitativo y eficiente, instrumentando la financiación de las unidades sanitarias desde la esfera nacional hacia los puntos provinciales y, respectivamente, a los municipales a través del Ministerio de Salud Pública. Se registran en algunos casos, independencia presupuestaria del Estado Central.

En cuanto a las primeras normas en esta materia, nacen como un sistema. Sin embargo, si bien el Ministerio de Salud Pública fue la cuna de disposiciones en tecnología sanitaria, un estudio correlacional con las normas actuales muestra que el derrotero histórico no siguió esta inteligencia y las mismas se constituyen en focos aislados de regulación que intentaron captar a los fenómenos científicos tecnológicos sin tomar en cuenta normas anteriores ni el contexto en el que fueron dadas, provocando solapamiento de incumbencias, conflicto de normas y dejando lagunas jurídicas en los ordenamientos jurídicos vigentes.

\section{Reconocimiento}

Este trabajo se lleva adelante como parte de un Proyecto de Investigación acreditado por la Secretaría General de Ciencia y Técnica de Universidad Nacional del Nordeste, Corrientes, Argentina. 